\title{
Isolation and Characterization of Saponin-Producing Fungal Endophytes from Aralia elata in Northeast China
}

\author{
Hao Wu, Hongyan Yang, Xiangling You and Yuhua Li * \\ College of Life Sciences/Daqing Bio-tech Research Institute, Northeast Forestry University, \\ Harbin 150040, Heilongjiang, China; E-Mails: mad_knight@163.com (H.W.); \\ cnyanghy@163.com (H.Y.); yxiangling@yahoo.com (X.Y.) \\ * Author to whom correspondence should be addressed; E-Mail: lyhshen@126.com; \\ Tel./Fax: +86-451-8219-1733.
}

Received: 9 October 2012; in revised form: 2 November 2012 / Accepted: 5 November 2012 / Published: 30 November 2012

\begin{abstract}
The purpose of this study was to investigate the diversity of endophytic fungi of Aralia elata distributed in Northeast China as well as their capacity to produce saponins. Ninety-six strains of endophytic fungi were isolated, and polymerase chain reaction (PCR) and sequencing were employed to identify the isolates. The saponin concentrations of the culture filtrates of representative strains were measured. The agar diffusion method was used to test antimicrobial activity, while high-performance liquid chromatography (HPLC) was employed to identify the saponins produced by representative strains. Alternaria, Botryosphaeria, Camarosporium, Cryptosporiopsis, Diaporthe, Dictyochaeta, Penicillium, Fusarium, Nectria, Peniophora, Schizophyllum, Cladosporium and Trichoderma species were isolated in this study. Overall, $25 \%$ of the isolates belonged to Diaporthe (Diaporthe eres), and $12.5 \%$ belonged to Alternaria. The highest concentration of saponins was produced by G22 (2.049 $\mathrm{mg} / \mathrm{mL})$. According to the results of the phylogenetic analysis, G22 belonged to the genus Penicillium. The culture filtrate of G22 exhibited antibacterial activity against Staphylococcus aureus, and ginsenosides Re and Rb2 were detected in G22 culture filtrates by HPLC.
\end{abstract}

Keywords: endophytic fungi; saponin; Aralia elata; microbial diversity 


\section{Introduction}

Fungi that colonize the living internal tissues of plants without causing any immediate and overt negative effects, have been called endophytic fungi [1]. Endophytic fungi have been found to be ubiquitous within all types of plants [2-4], and to play an important role in the ecological community. Some endophytic fungi have been found to influence their host's growth [5], enhance stress resistance [6], degrade pollutants [7], and produce biologically active substances [8].

Some biologically active substances produced by plants, can also be derived from endophytes of host plants. The most famous substance of this class is taxol, a mitotic inhibitor used in cancer chemotherapy. This compound was originally produced by the yew tree, but it can also be produced by their endophytic fungi [9]. In addition, endophytic fungi isolated from $A$. indica can secrete azadirachtin A and B, which are used to repel insects [10].

Aralia elata, which is native to Asia, is a medicinal plant belonging to the family Araliaceae [11]. A. elata can produce saponins [12]. Saponins produced by medical plants have multiple therapeutic values, including being protective against cancer $[13,14]$ and acting as a therapeutic agent for hepatitis and gastric ulcer $[15,16]$. Endophytic fungi in A. elata have not been well studied. Previously, Paul et al. reported the presence of endophytic fungi in roots of $A$. elata cultivated in Korea from the Chungnam province. In their study, the authors tested the antifungal activity against plant pathogenic fungi. Twenty-four genera were characterized, and Strumella, Rhizopycnis and Entrophospora were the most abundant taxa. Four isolates of Pyrenochaeta, 1 isolated of Entrophospora and 1 unidenitified species were positive against 6 plant pathogenic fungi tested [11]. To the best of our knowledge, there are no reports on endophytic fungi isolated from wild A. elata grown in Northeast China. In the present study, we investigated the diversity of the endophytic fungi harbored in populations of $A$. elata widely distributed in the Xiaoxing'anling area (Heilongjiang, China). The saponin production and antimicrobial activity of typical strains were analyzed.

\section{Results and Discussion}

\subsection{Strains Identification and Phylogenetic Analysis}

Ninety-six strains were isolated. Genomic DNA was extracted and the 28S rDNA D1/D2 region was amplified and sequenced. The obtained sequences were compared with those in the GenBank database, and the results are shown in Table 1.

Table 1. Similarity between the isolates and closest species in GenBank.

\begin{tabular}{llll}
\hline Strain ID (Strains) & Accession no. & Closest species (Accession no.) & Coverage (\%) \\
\hline P17 (24) & JQ807963 & Fungal sp. (GU552516) & 100 \\
& & Diaporthe sp. (DQ377874) & 100 \\
& & Diaporthe eres (AF362565) & 100 \\
G44-1 (12) & \multirow{2}{*}{ JQ807974 } & Uncultured Alternaria (JF495245) & 100 \\
& & Uncultured Alternaria (JF449889) & 100 \\
& & Alternaria sp. (GU048607) & 100 \\
\hline
\end{tabular}


Table 1. Cont.

\begin{tabular}{|c|c|c|c|}
\hline Strain ID (Strains) & Accession no. & Closest species (Accession no.) & Coverage (\%) \\
\hline \multirow[t]{3}{*}{ G22 (8) } & JQ807910 & Penicillium sp. (GU048572) & 100 \\
\hline & & Penicillium sp. (DQ123664) & 100 \\
\hline & & Penicillium rivolii (GU033419) & 99.3 \\
\hline \multirow[t]{3}{*}{ G27 (8) } & JQ807969 & Cladosporium sp. (FJ790290) & 100 \\
\hline & & Cladosporium cladosporioides (AY213695) & 100 \\
\hline & & Passalora fulva (AB100653) & 100 \\
\hline \multirow[t]{3}{*}{ P6 (6) } & JQ807984 & Schizophyllum commune (AM269871) & 99.8 \\
\hline & & Schizophyllum commune (AB428351) & 99.8 \\
\hline & & Schizophyllum commune (AB363767) & 99.8 \\
\hline \multirow[t]{3}{*}{$\mathrm{P} 11(5)$} & JQ807967 & Uncultured Dictyochaeta (JF449595) & 99.3 \\
\hline & & Uncultured Dictyochaeta (JF449592) & 98.8 \\
\hline & & Dictyochaeta simplex (AF178559) & 98 \\
\hline \multirow[t]{3}{*}{$\mathrm{P} 18(5)$} & JQ807982 & Fungal sp. (GU552507) & 100 \\
\hline & & Camarosporium leucadendri (EU552106) & 99.7 \\
\hline & & Camarosporium brabeji (EU552105) & 99.7 \\
\hline \multirow[t]{2}{*}{ G3 (5) } & JQ807935 & Nectria haematococca (HM042416) & 100 \\
\hline & & Nectria haematococca (AB373719) & 100 \\
\hline \multirow[t]{3}{*}{ G41 (4) } & JQ807972 & Cladosporium cladosporioides (JN651416) & 100 \\
\hline & & Cladosporium silenes (JF770463) & 100 \\
\hline & & Uncultured Cladosporium (JF449832) & 100 \\
\hline \multirow[t]{3}{*}{ G46 (4) } & JQ807956 & Fusarium avenaceum (JN938913) & 100 \\
\hline & & Fusarium sp. (JF700486) & 100 \\
\hline & & Fusarium avenaceum (HM068325) & 100 \\
\hline \multirow[t]{3}{*}{ P37 (3) } & JQ807942 & Peniophora sp. (HM595610) & 99.8 \\
\hline & & Peniophoraceae sp. (AB576771) & 99.8 \\
\hline & & Peniophoraceae sp. (DQ094783) & 99.8 \\
\hline \multirow[t]{3}{*}{$\mathrm{P} 24(3)$} & JQ807922 & Uncultured soil fungus (JQ311518) & 99.5 \\
\hline & & Trichoderma parareesei (JN874490) & 99.3 \\
\hline & & Trichoderma sp. (AB373724) & 99.1 \\
\hline \multirow[t]{3}{*}{ P16 (3) } & JQ807976 & Uncultured Pleosporales (JF691161) & 100 \\
\hline & & Uncultured Epicoccum (JF449817) & 100 \\
\hline & & Uncultured Epicoccum (JF449816) & 100 \\
\hline \multirow[t]{3}{*}{ P36 (2) } & JQ807968 & Uncultured Cryptosporiopsis (JF519417) & 100 \\
\hline & & Uncultured Cryptosporiopsis (JF519423) & 99.8 \\
\hline & & Cryptosporiopsis ericae (AY853167) & 99.8 \\
\hline \multirow[t]{3}{*}{ G5 (2) } & JQ807983 & Fungal sp. (GU552486) & 100 \\
\hline & & Botryosphaeria dothidea (AB454278) & 100 \\
\hline & & Botryosphaeria dothidea (FJ755233) & 100 \\
\hline \multirow[t]{3}{*}{ G49 (1) } & JQ807918 & Uncultured soil fungus (EU691410) & 100 \\
\hline & & Uncultured soil fungus (EU691436) & 100 \\
\hline & & Uncultured fungus (EF639724) & 100 \\
\hline \multirow[t]{3}{*}{ G39 (1) } & JQ807981 & Corynespora smithii (GU323201) & 94.6 \\
\hline & & Fenestella fenestrate (GU205220) & 94.6 \\
\hline & & Phoma sp. (JF746158) & 94.3 \\
\hline
\end{tabular}


Figure 1. A $28 \mathrm{~S}$ rDNA sequence-based phylogenetic tree constructed using the neighbor-joining method. Scale bar represents $10 \%$ estimated sequence divergence. Numbers in the branches indicate bootstrap values (percentages for 1000 replicates).

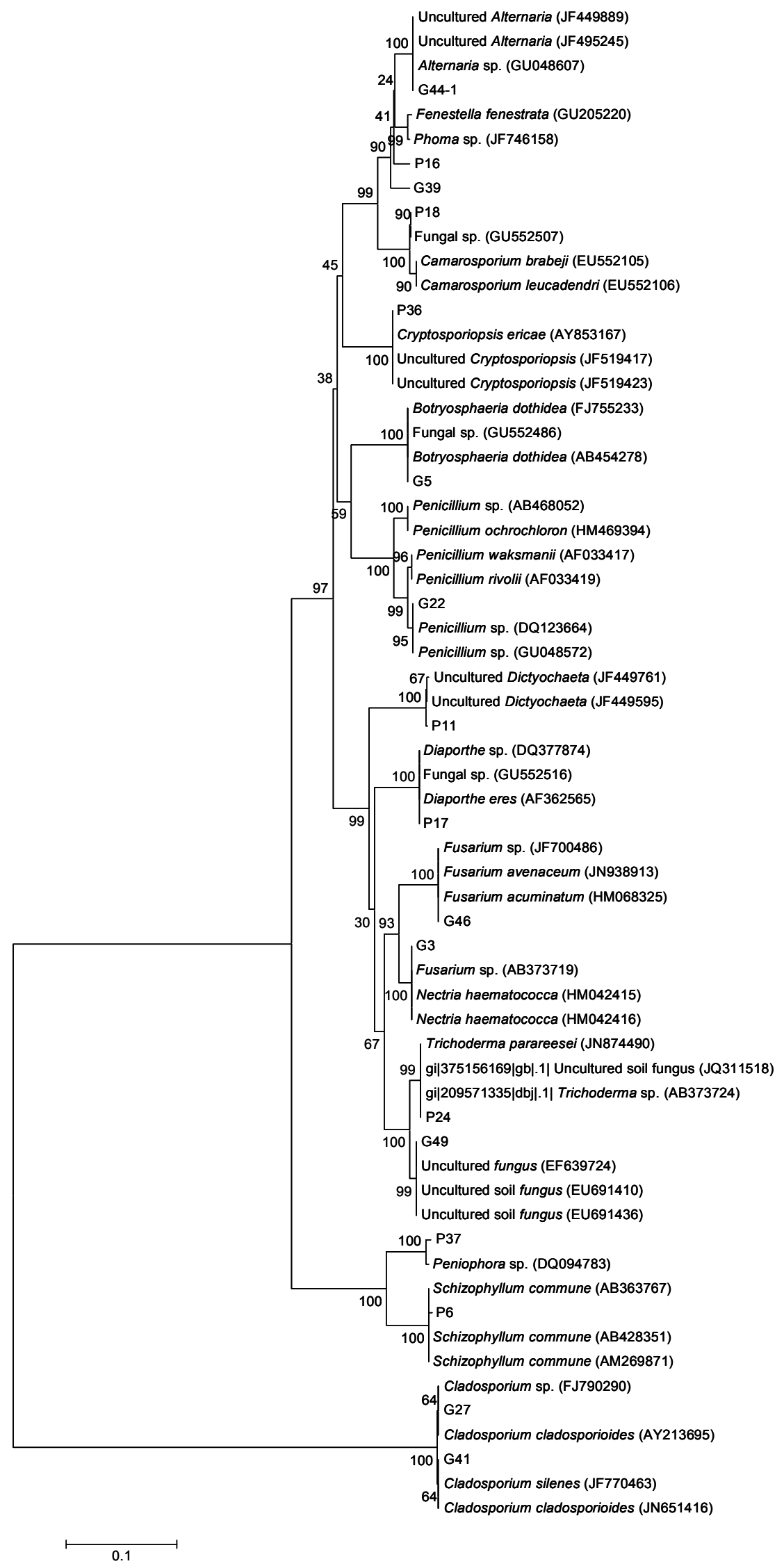


A phylogenetic tree built from the $28 \mathrm{~S}$ rDNA sequences is shown in Figure 1. From Table 1 and Figure 1, the identified fungi included: Alternaria, Botryosphaeria, Camarosporium, Cryptosporiopsis, Diaporthe, Dictyochaeta, Penicillium, Fusarium, Nectria, Peniophora, Schizophyllum, Cladosporium and Trichoderma. The most abundant genera were Diaporthe and Alternaria with 25 and 12.5\% of the total number of isolates, respectively. G49 was not identified because its sequence was significantly similar to unknown fungal sequences in the GenBank database.

Diaporthe and Altenaria were found to be the predominant genera, a finding that is different from previous studies showing that Strumella, Rhizopycnis and Entrophospora were the most abundant taxa in A. elata [11]. A possible reason for this discrepancy is that the A. elata specimens were derived from different regions [17].

Diaporthe are endophytic fungi that grow in several of plant species and have been shown to produced different secondary metabolites. For example Diaporthe sp. isolated from Espeletia sp. can inhibit the growth of Phytophthora infestans, a plant pathogen [18]. While Diaporthe sp. from Curcuma longa can convert curcumin into colorless hydroderivatives. Curcumin has a potent antioxidant effect. However, the distinct yellow color limits its use. This conversion may expand its application [19]. Diaporthe sp. P133, isolated from Pandanus amaryllifolius, can secrete benzopyranones, which inhibit a virulent strain of Mycobacterium tuberculosis [20]. Diaporthe phaseolorum isolated from mangrove forest can produce the antibacterial agent 3-hydroxypropionic acid [21]. A new varied species Alternaria alternata from the bark of 200-year-old Taxus cuspidate could produce taxoids of type III with the anti-neoplastic action [22]. Alternaria sp. isolated from Brassica juncea have demonstrated potential applications in biofuel feedback [23]. To the best of our knowledge, there are no reports concerning Diaporthe and Altenaria isolated from A. elata.

\subsection{Analysis of Triterpenoid Saponins and Antimicrobial Activity}

The concentration of triterpenoid saponin in the representative isolate from each group (Table 1) is shown in Table 2. The highest concentration of saponins was found in G22 $(2.049 \mathrm{mg} / \mathrm{mL})$, and this concentration is significantly higher than the level observed in P11 and P18 $(p<0.05)$. According to the results of phylogenetic analysis, G22 was identified as a Penicillium sp., P11 was identified as a Dictyochaeta sp., and P18 was identified as Camarosporium sp. The saponin concentrations among the strains of the same genus, such as G22 $(2.049 \mathrm{mg} / \mathrm{mL})$ and P23 $(0.049 \mathrm{mg} / \mathrm{mL})$, were significantly different $(p<0.05)$.

The growth-promoting factors and metabolites produced by endophytic fungi have been widely investigated and applied in both medicine and agriculture. The most notable substance produced by host endophytic fungi is taxol, a mitotic inhibitor used in cancer chemotherapy, and was originally produced by the yew tree [9]. Saponins produced by $A$. elata have multiple therapeutic values. The culture filtrates of endophytic fungi were analyzed to identify endophytes that produce triterpenoid saponins. It is well known that Penicillium is the source of penicillin, and recent studies show that endophytic Penicillium sp. also has the capacity to secrete anti-tumor substances [24,25] and gibberellin [26,27]. In this study, G22 (Penicillium sp.) has the high capacity to produce triterpenoid saponins. 
Table 2. Triterpenoid saponin production of the representative isolate from each group.

\begin{tabular}{ccl}
\hline Isolate ID & Mean \pm Stdev $(\mathbf{m g} / \mathbf{m L})(\boldsymbol{p}<\mathbf{0 . 0 5})$ & \multicolumn{1}{c}{ Expected species } \\
\hline G22 & $2.049 \pm 0.044 \mathrm{a}$ & Penicillium $\mathrm{sp}$. \\
$\mathrm{P} 11$ & $0.162 \pm 0.004 \mathrm{~b}$ & Dictyochaeta $\mathrm{sp}$. \\
P18 & $0.156 \pm 0.006 \mathrm{~b}$ & Camarosporium leucadendri \\
G27 & $0.131 \pm 0.003 \mathrm{c}$ & Cladosporium sp. \\
P6 & $0.120 \pm 0.003 \mathrm{~cd}$ & Schizophyllum commune \\
G49 & $0.113 \pm 0.005 \mathrm{~cd}$ & Uncultured soil fungus \\
P37 & $0.109 \pm 0.006 \mathrm{~d}$ & Peniophora sp. \\
P17 & $0.084 \pm 0.005 \mathrm{e}$ & Diaporthe $\mathrm{sp}$. \\
P16 & $0.066 \pm 0.005 \mathrm{ef}$ & Epicoccum sp. \\
G41 & $0.066 \pm 0.006 \mathrm{efg}$ & Cladosporium cladosporioides \\
G46 & $0.065 \pm 0.003 \mathrm{efg}$ & Fusarium avenaceum \\
P24 & $0.060 \pm 0.006 \mathrm{fg}$ & Trichoderma parareesei \\
G44-1 & $0.059 \pm 0.002 \mathrm{fg}$ & Alternaria sp. \\
G39 & $0.048 \pm 0.001 \mathrm{gh}$ & Corynespora smithii \\
G5 & $0.038 \pm 0.004 \mathrm{~h}$ & Botryosphaeria dothidea \\
G3 & $0.032 \pm 0.005 \mathrm{~h}$ & Nectria haematococca \\
P36 & $0.030 \pm 0.002 \mathrm{~h}$ & Cryptosporiopsis sp. \\
\hline
\end{tabular}

From Table 3, G22 exhibited antibacterial activity against Gram-positive bacterium Staphylococcus aureus ACCC10499. P11 inhibited the growth of S. aureus ACCC10499, Rhizoctonia solani ACCC36233 and Fusarium sporotrichioides. At the same time, it expressed strong inhibition to Klebsiella pneumoniae ACCC10498. P18 also showed inhibition to K. pneumoniae ACCC10498.

Table 3. Antimicrobial activity of representative endophytic fungi strains.

\begin{tabular}{lccc}
\hline \multirow{2}{*}{ Test strains } & \multicolumn{3}{c}{ Representative endophytic strains } \\
\cline { 2 - 4 } & G22 & P11 & P18 \\
\hline Staphylococcus aureus ACCC10499 & ++ & + & - \\
Bacillus subtitis ACCC10243 & - & - & - \\
Klebsiella pneumoniae ACCC10498 & - & ++ & + \\
Pseudomonas aeruginosa ACCC10500 & - & - & - \\
Phytophthora cactorum ACCC36421 & - & - & - \\
Rhizoctonia solani ACCC36233 & - & + & - \\
Aspergillus niger ACCC30005 & - & - & - \\
Fusarium sporotrichioides & - & + & - \\
\hline
\end{tabular}

(-) no inhibition; (+) presence of a zone of growth inhibition +++ width of growth inhibition zone $>10 \mathrm{~mm}$, $++5-10 \mathrm{~mm},+1-5 \mathrm{~mm}$.

\subsection{Ginsenosides Analyses}

To further analyze the composition of saponins, the culture filtrates of G22, P11, and P18, underwent HPLC. As a reference, eight ginsenoside standards were also analyzed. According to the spectra, which are shown in Figure 2, G22, P11 and P18 all produced detectable concentrations of 
saponins. Ginsenoside Rb2 was detected in the G22, P11, and P18 culture filtrates. More peaks were observed in the G22 culture filtrate. Ginsenoside Re was also detected in the G22 culture filtrate. These results indicate that the three strains have the capacity to produce ginsenosides, especially for G22. Additionally, G22 inhibited S. aureus growth (Table 3). These results indicate that G22 has a great potential for the further detailed study.

Figure 2. High-performance liquid chromatography (HPLC) spectra of the ginsenoside standards and the culture filtrates of the representative strains. 1, Rg1; 2, Re; 3, Rf; 4, Rb1; 5, Rc; 6, Rb2; 7, Rb3; 8, Rd.
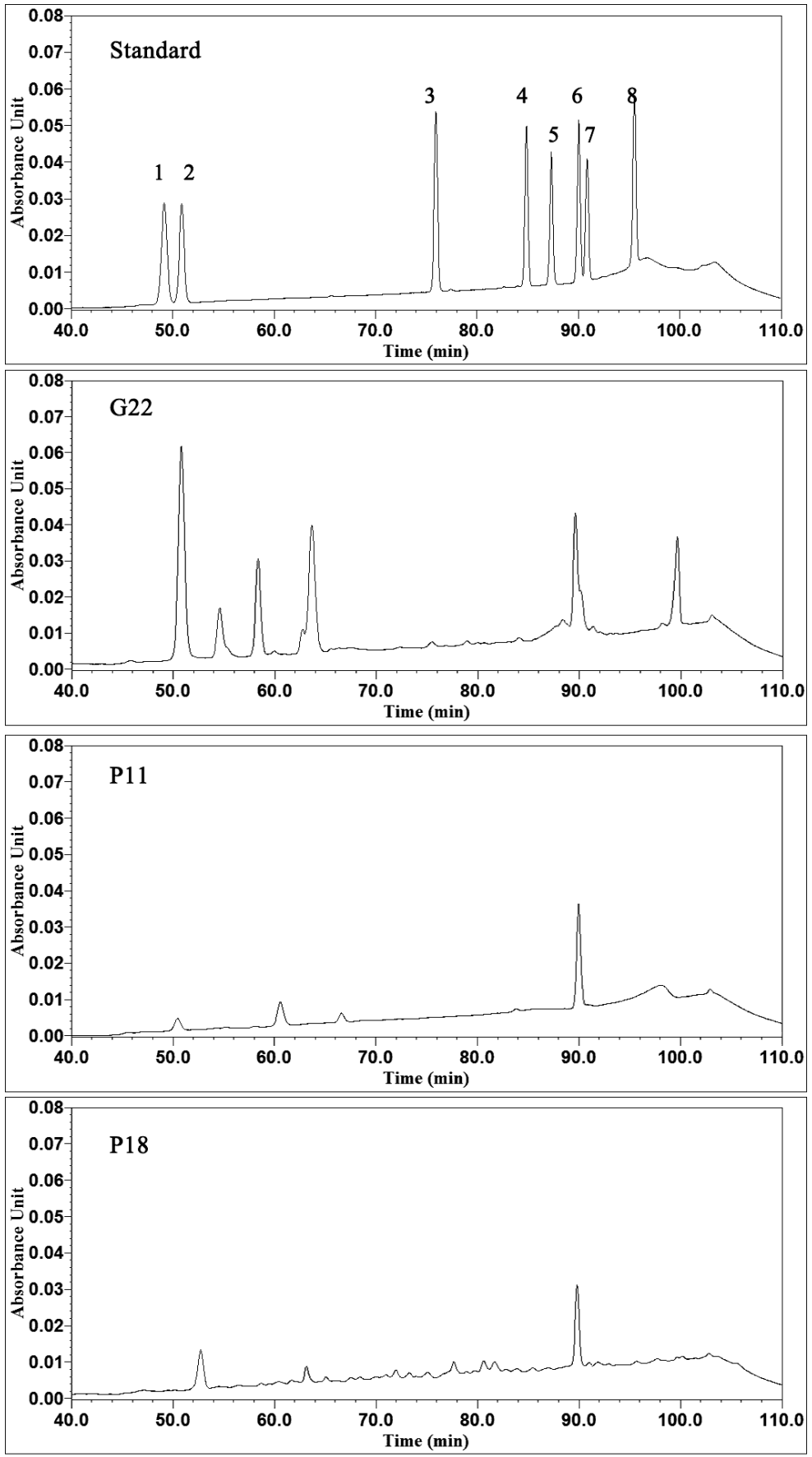


\section{Experimental Section}

\subsection{Sampling and Isolation}

Wild $A$. elata plants (5 years old) were sampled from the Xiaoxing'anling area in Northeast China. The $A$. elata roots were immediately placed in sterile plastic bags and stored at $4{ }^{\circ} \mathrm{C}$. The endophytes were isolated within $48 \mathrm{~h}$ of collection. Before disinfection, the plant samples were thoroughly washed under running tap water for $10 \mathrm{~h}$. The roots were surface-disinfected with $70 \%(v / v)$ ethanol for $0.5-1 \mathrm{~min}, 5 \% \mathrm{NaOCl}$ for $5-10 \mathrm{~min}, 70 \%(v / v)$ ethanol for $0.5-1 \mathrm{~min}$ and burning for $10-30 \mathrm{~s}$. The samples were subsequently rinsed with sterile water, and the outer tissue was removed with a sterile scalpel. Small pieces $(0.5 \times 0.5 \mathrm{~cm})$ of $A$. elata were placed in Petri dishes containing malt extract agar (Oxoid-Unipath Ltd., Hampshire, UK), Czapeck agar (Oxoid-Unipath Ltd., Hampshire, UK), or potato dextrose agar (Oxoid-Unipath Ltd., Hampshire, UK), and were incubated at $28{ }^{\circ} \mathrm{C}$ for seven days. Following the incubation, single colonies of distinctive morphotypes were isolated on the basis of their morphological characteristics and appearance. The colonies were subsequently re-isolated by plating on PDA and incubating at $28{ }^{\circ} \mathrm{C}$ for $24-48 \mathrm{~h}$ to obtain pure cultures. All of the isolates were vacuum freeze-dried and deposited in the collection of the College of Life Sciences, Northeast Forestry University.

\subsection{DNA Extraction and PCR Amplification of the $28 S$ rRNA Gene}

Fungal genomic DNA was extracted using the EZNA Fungal DNA Mini Kit (OMEGA, USA) according to the manufacturer's instructions. The $50 \mu \mathrm{L}$ PCR mixtures contained $15 \mathrm{ng}$ of template DNA, $1 \times$ PCR buffer $\left(\mathrm{Mg}^{2+}\right.$ free), $0.16 \mathrm{mM}$ of each dNTP, $1.5 \mathrm{mM} \mathrm{MgCl}_{2}, 0.45 \mu \mathrm{M}$ of each primer, and $1 \mathrm{U}$ of Takara rTaq DNA polymerase (Takara, Japan). The primers employed for the amplification of the D1/D2 region of the fungal 28S rRNA gene were NL1 (5'-GCATATCAATAAGCGGAGG AAAAG-3') and NL4 (5'-GGTCCGTGTTTCAAGACGG-3') [28]. The thermocycling program consisted of initial DNA denaturation at $95{ }^{\circ} \mathrm{C}$ for $5 \mathrm{~min}$ followed by 30 cycles of denaturation at $95{ }^{\circ} \mathrm{C}$ for $1 \mathrm{~min}$, annealing at $52{ }^{\circ} \mathrm{C}$ for $45 \mathrm{~s}$, and elongation at $72{ }^{\circ} \mathrm{C}$ for $1 \mathrm{~min} 30 \mathrm{~s}$, ending with a final elongation step at $72{ }^{\circ} \mathrm{C}$ for $6 \mathrm{~min}$ [29].

The PCR amplification products were separated by electrophoresis through $1 \%(w / v)$ agarose gels, stained with ethidium bromide and visually examined under UV light. The PCR products were purified using the Agarose Gel DNA Extraction Kit (Takara, Japan) and sequenced by Sangon Biotech (Shanghai, China).

\subsection{Phylogenetic Analysis and Nucleotide Sequence Accession Numbers}

The sequences generated in this study were compared with those in GenBank [30]; those sequences with $\geq 99 \%$ similarity to the $28 \mathrm{~S}$ rDNA D1/D2 regions (approximately $600 \mathrm{bp}$ ) were considered to belong to identical genera and were included in the alignment. A neighbor-joining tree was constructed using MEGA 5.0 software [31]. The number of bootstrap replications was 1000 . The sequences were deposited in GenBank under the accession numbers listed in Table 1. 


\subsection{Determination of Triterpenoid Saponins}

Each isolate was inoculated into $100 \mathrm{~mL}$ of PDA liquid medium (250 mL flask) and stirred at $150 \mathrm{rpm}$ at $28{ }^{\circ} \mathrm{C}$ for two weeks. After ultrasonication, the supernatant was separated from the cell debris by centrifugation at $4000 \times g$ for $20 \mathrm{~min}$. A $20 \mathrm{~mL}$ aliquot of the supernatant was poured into a $50 \mathrm{~mL}$ centrifuge tube (Corning Inc., Corning, NY, USA), and $20 \mathrm{~mL}$ of ethyl acetate was added to the same tube. After mixing, ultrasonication and incubation for $5 \mathrm{~min}, 5 \mathrm{~mL}$ of the supernatant was evaporated to dryness under a vacuum at $50{ }^{\circ} \mathrm{C}$. The residue was dissolved in $2 \mathrm{~mL}$ of methanol. The methanol solutions were centrifuged at $4000 \times g$ for $10 \mathrm{~min}$, and the supernatants were used for subsequent analysis of the total saponin content.

The measurement of the total extracted saponins was based on a color reaction of the acid-hydrolysis products of the saponins (i.e., sapogenins) with vanillin. In total, $5 \mathrm{~mL}$ of the supernatant was added to a test tube and evaporated at $60{ }^{\circ} \mathrm{C}$ in a water bath. The residue was dissolved in $0.2 \mathrm{~mL}$ of $5 \%$ vanillin, mixed with $0.8 \mathrm{~mL}$ of perchloric acid, incubated in a $60{ }^{\circ} \mathrm{C}$ water bath for $15 \mathrm{~min}$ and quickly cooled in ice water. The concentration $(\mathrm{mg} / \mathrm{mL})$ of saponins in the reaction sample was determined using a spectrophotometer at $560 \mathrm{~nm}$ and comparing the readings against a calibration curve established with an oleanolic acid standard (National Institute for the Control of Pharmaceutical and Biological Products, Beijing, China) [32].

\subsection{Antimicrobial Activity of the Representative Strains}

The antimicrobial activity of typical strains after 14-day cultivation against 8 microorganisms (listed in Table 3) was assessed by the agar diffusion method [33]. Three $6 \mathrm{~mm}$ wells were made in each disk. With the exception of Fusarium sporotrichioides (isolated in our lab), the strains were purchased from the Agricultural Culture Collection of China (ACCC). Streptomycin sulfate (5 mg/well), amoxicillin (5 mg/well) and itraconazole $(4.4 \mathrm{mg} /$ well) were used as positive antimicrobial controls. The activity of the extracts was estimated from the diameter $(\mathrm{mm})$ of the zone of inhibition.

\subsection{Ginsenosides Analyses}

One hundred milliliters of ethyl acetate was added to $100 \mathrm{~mL}$ liquid culture. Following $30 \mathrm{~min}$ of agitation at $160 \mathrm{rpm}$ and ultrasonication at $50{ }^{\circ} \mathrm{C}$, the supernatant was separated from the cell debris by centrifugation at $4000 \times \mathrm{g}$ for $30 \mathrm{~min}$. After evaporation, the pellet was dissolved in $5 \mathrm{~mL}$ of methanol, followed by filtration through a SepPak C-18 Cartridge (Waters, Milford, MA, USA). The following water/acetonitrile gradient system was employed during HPLC analysis: 0 min, 18\% acetonitrile and $82 \%$ water; $40 \mathrm{~min}, 18 \%$ acetonitrile and $82 \%$ water; $50 \mathrm{~min}, 22 \%$ acetonitrile and $78 \%$ water; $70 \mathrm{~min}$, $28 \%$ acetonitrile and $72 \%$ water; $100 \mathrm{~min}, 38 \%$ acetonitrile and $62 \%$ water; and $110 \mathrm{~min}, 18 \%$ acetonitrile and $82 \%$ water.

\section{Conclusions}

This study focused on the diversity of endophytic fungi from A. elata in Northeast China for the first time. Ninety-six strains were isolated. They were belonged to 12 genus respectively. The most 
abundant genera were Diaporthe and Alteraria represented by 25 and $12.5 \%$ of the isolates respectively. The analysis from saponins showed that many isolated fungi had the capacity to produce saponins. The highest concentration of saponins was found in G22 (Penicillium sp., $2.049 \mathrm{mg} / \mathrm{mL}$ ), Ginsenoside Rb2 and Re were detected in the G22.This result indicate that G22 has the capacity to produce ginsenosides and consequently has application potentials.

\section{Acknowledgments}

This study was supported by the Fund of "The National Forestry Public Welfare Industry Targeted Research Fund" (20100400703) and "The Daqing Technology Innovation Program" (SCX2010-08, SCYH-2011-95) and "The Daqing High-Tech Zone Special Fund” (DQGX10ZS006).

\section{References}

1. Huang, W.Y.; Cai, Y.Z.; Hyde, K.D.; Corke, H.; Sun, M. Biodiversity of endophytic fungi associated with 29 traditional Chinese medicinal plants. Fungal Divers 2008, 33, 61-75.

2. Hyde, K.D.; Soytong, K. The fungal endophyte dilemma. Fungal Divers 2008, 33, 163-173.

3. Koukol, O. New species of Chalara occupying coniferous needles. Fungal Divers 2011, 49, 75-91.

4. Li, H.; Shen, M.; Zhou, Z.; Li, T.; Wei, Y.; Lin, L. Diversity and cold adaptation of endophytic fungi from five dominant plant species collected from the Baima Snow Mountain, Southwest China. Fungal Divers 2012, 54, 79-86.

5. Alberton, O.; Kuyper, T.W.; Summerbell, R.C. Dark septate root endophytic fungi increase growth of Scots pine seedlings under elevated $\mathrm{CO}_{2}$ through enhanced nitrogen use efficiency Plant Soil 2010, 328, 459-470.

6. Saikkonen, K.; Saari, S.; Helander, M. Defensive mutualism between plants and endophytic fungi? Fungal Divers 2010, 41, 101-113.

7. Osono, T.; Hirose, D. Ecology of endophytic fungi associated with leaf litter decomposition. In Applied Mycology; Rai, M., Bridge, P.D., Eds.; CAB International: Cambridge, MA, USA, 2009; pp. 92-109.

8. Schulz, B.; Boyle, C.; Draeger, S.; Römmert, A.; Krohn, K. Endophyticfungi: A source of novel biologically active secondary metabolites. Mycol. Res. 2002, 106, 996-1004.

9. Rivera-Orduña, F.N.; Suarez-sanchez, R.A.; Flores-Bustamante, Z.R.; Gracida-Rodriguez, J.N.; Flores-Cotera, L.B. Diversity of endophytic fungi of Taxus globosa (Mexican yew). Fungal Divers 2011, 47, 65-74.

10. Kusari, S.; Verma, V.C.; Lamshoeft, M.; Spiteller, M. An endophytic fungus from Azadirachta indica A. Juss. that produces azadirachtin. World J. Microbiol. Biotechnol. 2012, 28, 1287-1294.

11. Paul, N.C.; Kim, W.K.; Woo, S.K.; Park, S.; Yu, S.H. Fungal endophytes in roots of Aralia species and their antifungal activity. Plant Pathol. J. 2007, 23, 287-294.

12. Sakai, S.; Katsumata, M.; Satoh, Y.; Nagasao, M.; Miyakoshi, M.; Ida, Y.; Shoji, J. Oleanolic acid saponins from root bark of Aralia elata. Phytochemistry 1994, 35, 1319-1324. 
13. Mujoo, K.; Haridas, V.; Hoffmann, J.J.; Wächter, G.A.; Hutter, L.K.; Lu, Y.; Blake, M.E.; Jayatilake, G.S.; Bailey, D.; Mills, G.B.; et al. Triterpenoid saponins from Acacia victoriae (Bentham) decrease tumor cell proliferation and induce apoptosis. Cancer Res. 2001, 61, 5486-5490.

14. Fujimoto, J.; Sakaguchi, H.; Alki, I.; Toyoki, H.; Khatun, S.; Tamaya, T. Inhibitory effect of ginsenoside- $\mathrm{Rb} 2$ on invasiveness of uterine endometrial cancer cells to the basement membrane. Eur. J. Gynaecol. Oncol. 2001, 22, 339-341.

15. Zhao, Y.-L.; Cai, G.-M.; Hong, X.; Shan, L.-M.; Xiao, X.-H. Anti-hepatitis B virus activities of triterpenoid saponin compound from Potentilla anserine L. Phytomedicine 2008, 15, 253-258.

16. Sen, S.; Chakraborty, R.; De, B.; Mazumder, J. Plants and phytochemicals for peptic ulcer: An overview. Pharmacognosy Rev. 2009, 3, 270-279.

17. Davis, E.C.; Shaw, A.J. Biogeographic and phylogenetic patterns in diversity of liverwort-associated endophytes. Am. J. Bot. 2008, 95, 914-924.

18. Prada, H.; Avila, L.; Sierra, R.; Bernal, A.; Restrepo, S. Morphological and molecular characterization of the antagonistic interaction between the endophyte Diaporthe sp. isolated from frailejon (Espeletia sp.) and the plant pathogen Phytophthora infestans. Rev. Iberoam. Micol. 2009, 26, 198-201.

19. Maehara, S.; Ikeda, M.; Haraguchi, H.; Kitamura, C.; Nagoe, T.; Ohashi, K.; Shibuya, H. Microbial conversion of curcumin into colorless hydroderivatives by the endophytic fungus Diaporthe sp. associated with Curcuma longa. Chem. Pharm. Bull. 2011, 59, 1042-1044.

20. Bungihan, M.E.; Tan, M.A.; Kitajima, M.; Kogure, N.; Franzblau, S.G.; Dela Cruz, T.E.; Takayama, H.; Nonato, M.G. Bioactive metabolites of Diaporthe sp. P133, an endophytic fungus isolated from Pandanus amaryllifolius. J. Nat. Med. 2011, 65, 606-609.

21. Sebastianes, F.L.S.; Cabedo, N.; Aouad, N.E.; Valente, A.M.M.P.; Lacava, P.T.; Azevedo, J.L.; Pizzirani-Kleiner, A.A.; Cortes, D. 3-Hydroxypropionic acid as an antibacteria agent from endophytic fungi Diaporthe phaseolorum. Curr. Microbiol. 2012, doi:10.1007/s002834-012-0206-4.

22. Xiang, Y.; Lu, A.; Wu, W. Identification of Taxus cuspidata sieb. et Zucc. endophytic fungi-new species, species known and their metabolite. J. For. Res. 2003, 14, 290-294.

23. Dey, P.; Banerjee, J.; Maiti, M.K. Comparative lipid profiling of two endophytic fungal isolates-Colletotrichum sp. and Alternaria sp. having potential utilities as biodiesel feedstock. Bioresource Technol. 2011, 102, 5815-5823.

24. Chen, G.; Zhu, Y.; Wang, H.Z.; Wang, S.J.; Zhang, R.Q. The metabolites of a mangrove endophytic fungus, Penicillium thomi. J. Asian Nat. Prod. Res. 2007, 9, 159-164.

25. Guo, Z.; Cheng, F.; Zou, K.; Wang, J.; She, Z.; Lin, Y. Secondary metabolites from the mangrove endophytic fungus Penicillium sp. (SBE-8). Nat. Prod. Commun. 2009, 4, 1481-1483.

26. Hamayun, M.; Khan, S.A.; Iqbal, I.; Ahmad, B.; Lee, I.J. Isolation of a gibberellin-producing fungus (Penicillium sp. MH7) and growth promotion of Crown daisy (Chrysanthemum coronarium). J. Microbiol. Biotechnol. 2010, 20, 202-207.

27. Ahmad, N.; Hamayun, M.; Khan, S.A.; Khan, A.L.; Lee, I.J.; Shin, D.H. Gibberellin-producing endophytic fungi isolated from Monochoria vaginalis. J. Microbiol. Biotechnol. 2011, 20, 1744-1749. 
28. Redecke, D. Specific PCR primers to identify arbuscular mycorrhizal fungi within colonized roots. Mycorrhiza 2000, 10, 73-80.

29. Yang, H.; Wu, H.; Wang, X.; Cui, Z.; Li, Y. Selection and characteristics of a switchgrass-colonizing microbial community to produce extracellular cellulases and xylanases. Bioresour. Technol. 2011, 102, 3546-3550.

30. GenBank. Basic Local Alignment Search Tool. Available online: http://blast.ncbi.nlm.nih.gov/Blast.cgi (accessed on 12 June 2012).

31. Tamura, K.; Peterson, D.; Peterson, N.; Stecher, G.; Nei, M.; Kumar, S. MEGA5: Molecular evolutionary genetics analysis using maximum likehood, evolutionary distance, and maximum parsimony methods. Mol. Biol. Evol. 2011, 28, 2731-2739.

32. Liu, H.G.; Li, T.; Zhao, Y.L.; Zhang, J.; Wang, Y.Z. Determination of some metabolites of Cordyceps sobolifera. Afr. J. Microbiol. Res. 2011, 5, 5518-5522.

33. Hormazabal, E.; Piontelli, E. Endophytic fungi from chilean native gymnosperms: Antimicrobial activity against human and phytopathogenic fungi. World J. Microbiol. Biotechnol. 2009, 25, 813-819.

(C) 2012 by the authors; licensee MDPI, Basel, Switzerland. This article is an open access article distributed under the terms and conditions of the Creative Commons Attribution license (http://creativecommons.org/licenses/by/3.0/). 